\title{
Nothing Ventured, Nothing Gained? Navigating Disease-Modifying Treatment in Multiple Sclerosis
}

Keywords: Multiple sclerosis, Disease-modifying treatments, NEDA

In this issue of the CJNS, Freedman et al (2018) provide a detailed guide to the dual potential approaches to diseasemodifying therapy (DMT) choice in relapsing multiple sclerosis (MS). ${ }^{1}$ The classic, and as of yet unresolved, debate is typically between an induction and escalation approach. With the introduction of natalizumab in 2004 and subsequent additions to our arsenal, the greater potency of agents has brought with them considerable concern about potential rare, but serious, adverse events.

In recent years, MS DMTs potentially offer upwards of $70+\%$ relapse reduction rates (vs. $\sim 30 \%$ with first-generation injectables), reduced sustained accumulation of disability, and, in addition to marked reduction in new MRI lesions, the possibility of reduced rates of brain atrophy. ${ }^{2-7}$ This is in tandem with our gains in the knowledge that brain volume, and seemingly normalappearing white and grey matter, start to undergo damage and degeneration in the earliest days of the disease. ${ }^{8}$ In addition, in recent years, MS treatment goals have been centred around the concept of "no evidence of disease activity, or NEDA", namely no clinical or conventional radiological evidence of activity (NEDA-3 if no evidence of new T2/gadolinium lesions and NEDA-4 with the addition of no evidence of brain atrophy). ${ }^{9}$ Those who meet NEDA criteria appear to be at a relatively lower risk of disability accumulation, as NEDA rates at 2 years have shown a $78 \%$ positive predictive value of no progression at year 7. ${ }^{9}$ That being said, claims about NEDA remain controversial as several studies have not shown NEDA to predict some of the more progressive elements of disease advancement. ${ }^{9}$

One argument is that an "as-needed" escalation approach to DMTs, starting with the gentlest agents first, will abrogate adverse events, particularly in a young adult who may live another 40-50 years. However, such a simplistic view does not take into account disease behaviour before DMT selection, which may predict the severity of MS activity to come, nor does it address how and when the decision to escalate should occur. The opposite argument is that, like our colleagues in rheumatology, we should choose from among the most potent agents at the start with the goal of eliminating all signs of disease activity (i.e., a NEDA-like state), which may result in less MS-associated disability in the long term. ${ }^{10,11}$ Furthermore, it might be better to use these agents while the patient is young and relatively disability-free rather than wait. Newer therapies are more effective than the first-generation therapies, yet NEDA remains an elusive goal, with only $48 \%$ of ocrelizumab trial patients achieving NEDA at year 2, whereas at 5 years in alemtuzumab trial patients, the proportion was between $58 \%$ and $62 \% .^{9,12}$ Reserving these therapies for early use in a select group of patients may be a dis-service to other relapsing remitting MS (RRMS) patients with an uncertain prognosis.

With respect to second- and third-line agents, and even some firstline agents, we have had to acknowledge and become well versed in a host of new potential serious adverse events, with the need for much closer monitoring and diligence. It is now well known that the risk of progressive multifocal leukoencephalopathy (PML) with natalizumab can be significant and, to some degree, predictable, based on risk factors of time on drug (>24 months), John Cunningham virus (JCV) serology positivity and titre, and past immunosuppressive exposure. In a small percentage of patients, dimethyl fumarate (DMF) is associated with a potential drop in lymphocytes, rarely grade 3 or 4 in severity. ${ }^{13,14}$ Left unaddressed, this exposes a patient to both common and opportunistic infections, including tuberculosis (TB) reactivation and PML. In fact DMF has been associated with five cases of PML, although in most cases, unaddressed chronic severe lymphopaenia and older age appeared to be common factors. ${ }^{15}$ Fingolimod is associated with rare but potentially serious cardiac complications including symptomatic bradycardia and long QT syndrome. ${ }^{2,3}$ In addition, although disseminated zoster is likely avoidable with proper screening for varicella zoster virus (VZV) immune status, zoster eruptions are not uncommon. Most concerning are the ten and counting known cases of PML in fingolimod users, and without any obvious common predictable features to guide care providers. ${ }^{15}$ Alemtuzumab is linked to moderate infusion reactions and has a $30+\%$ risk of thyroid dysfunction, a $2 \%$ risk of idiopathic thrombocytopenic purpura (ITP) based on clinical trials, and rare glomerular nephropathy syndromes $(0.3 \%) .{ }^{4,5}$ Furthermore, these adverse events can occur up to 4 years after dosing, necessitating monthly lab monitoring for a minimum of 5-6 years. ${ }^{4,5}$ There are also multiple cases of Listeria monocytogenes infections, including meningitis, associated with alemtuzumab, probably in those who have ingested unpasteurised dairy products. ${ }^{16}$ Ocrelizumab is associated with infusion reactions, infections including zoster, and an as of yet to be clarified potential association with malignancy, particularly breast cancer. ${ }^{6}$ These risks can be mitigated, and the risk of future disability with inadequately treated MS cannot be underestimated.

The intricacies of choosing the best therapy for MS patients is unlikely to boil down to a simple, one size fits all guideline. Such choices must take into account the long-term benefit of avoiding disease activity early, aspiring to NEDA, as well as the individual patient's goals and risk tolerance. At the very least, such decisions and oversight should ideally be under the care of an MS speciality clinic/centre to ensure optimal patient disease monitoring, as well as

Received June 29, 2018. Date Of Acceptance July 4, 2018. 
appropriate access, staffing, and expertise to move quickly on potential adverse events.

\section{Statement of Authorship}

Both authors contributed equally to the content of this manuscript.

\section{Disclosure}

JB reports grants from Novartis, grants from Sanofi Genzyme, grants from EMD Serono, and personal fees from Novartis, outside the submitted work. AT reports grants and personal fees from Biogen, grants and personal fees from Roche, grants and personal fees from Sanofi Genzyme, grants and personal fees from Teva, grants and personal fees from Novartis, grants and personal fees from Chugai, and personal fees from EMD Serono, outside the submitted work.

Jodie M. Burton

Department of Clinical Neurosciences Hotchkiss Brain Institute, University of Calgary, Calgary Alberta Canada

Anthony Traboulsee Department of Medicine, Division of Neurology University of British Columbia, Vancouver, Canada

Correspondence to: Jodie M. Burton, Department of Clinical Neurosciences, University of Calgary, 3330 Hospital Drive NW, Calgary, AB, Canada T2N 4N1. Email: jodie.burton@alberta healthservices.ca

\section{REFERENCES}

1. Freedman MS, Selchen D, Prat A, Giacomini PS. Managing multiple sclerosis: treatment initiation, modification, and sequencing. Can J Neurol Sci. 2018;45(5):489-503.

2. Kappos L, Radue EW, O'Connor P, et al. A placebo-controlled trial of oral fingolimod in relapsing multiple sclerosis. N Engl J Med. 2010;362(5):387-401.
3. Cohen JA, Barkhof F, Comi G, et al. Oral fingolimod or intramuscular interferon for relapsing multiple sclerosis. N Engl J Med. 2010;362(5):402-15.

4. Cohen JA, Coles AJ, Arnold DL, et al. Alemtuzumab versus interferon beta 1a as first-line treatment for patients with relapsingremitting multiple sclerosis: a randomised controlled phase 3 trial. Lancet. 2012;380:1819-28.

5. Coles AJ, Twyman CL, Arnold DL, et al. Alemtuzumab for patients with relapsing multiple sclerosis after disease-modifying therapy: a randomised controlled phase 3 trial. Lancet. 2012;380:1829-39.

6. Hauser SL, Bar-Or A, Comi G, et al. Ocrelizumab versus interferon beta-1a in relapsing multiple sclerosis. $\mathrm{N}$ Engl $\mathrm{J}$ Med. 2017;376:221-34.

7. Fogarty E, Schmitz S, Turbidy N, Walsh C, Barry M. Comparative efficacy of disease-modifying therapies for patients with relapsing remitting multiple sclerosis: systematic review and network meta-analysis. Mult Scler Relat Disord. 2016;9:23-30.

8. Simon J. Very early MS - insights from MRI. Mult Scler. 2012;18 (10):1372-6.

9. Parks NE, Flanagan EP, Lucchinetti CF, Wingerchuk DM. NEDA treatment target? No evident disease activity as an actionable outcome in practice. J Neurol Sci. 2017;383:31-4.

10. Giovanonni G. Multiple sclerosis should be treated using a stepdown strategy rather than a step-up strategy-YES. Mult Scler. 2016;22(11):1397-400.

11. Sormani MP, Arnold DL, De Stefano N. Treatment effect on brain atrophy correlates with treatment effect on disability in multiple sclerosis. Ann Neurol. 2014;75:43-9.

12. Coles AJ, Cohen JA, Fox EJ, et al. Alemtuzumab CARE-MS II 5year follow-up: efficacy and safety findings. Neurology. 2017;89 (11):1117-26.

13. Gold R, Kappos L, Arnold DL, et al. Placebo-controlled phase 3 study of oral BG-12 for relapsing multiple sclerosis. N Engl J Med. 2012;367(12):1098-107.

14. Fox RJ, Miller DH, Phillips JT, et al. Placebo-controlled phase 3 study of oral BG-12 or glatiramer in multiple sclerosis. N Engl J Med. 2012;367(12):1087-97.

15. Berger JR. Classifying PML risk with disease modifying therapies. Mult Scler Relat Disord. 2017;12:59-63.

16. Holmoy T, von der Lippe H, Leegaard TM. Listeria monocytogenes infection associated with alemtuzumab - a case for better preventive strategies. BMC Neurol 2017; 17: 65. 